Louisiana State University

LSU Digital Commons

$6-7-2020$

\title{
The influence of Au substitution and hydrostatic pressure on the phase transitions and magnetocaloric properties of $\mathrm{MnCoGe}$ alloys
}

Jing Han Chen

Louisiana State University

Aaron Trigg

Louisiana State University

Tej Poudel Chhetri

Louisiana State University

David P. Young

Louisiana State University

Igor Dubenko

Southern Illinois University Carbondale

See next page for additional authors

Follow this and additional works at: https://digitalcommons.Isu.edu/physics_astronomy_pubs

\section{Recommended Citation}

Chen, J., Trigg, A., Poudel Chhetri, T., Young, D., Dubenko, I., Ali, N., \& Stadler, S. (2020). The influence of Au substitution and hydrostatic pressure on the phase transitions and magnetocaloric properties of MnCoGe alloys. Journal of Applied Physics, 127 (21) https://doi.org/10.1063/5.0007172 


\section{Authors}

Jing Han Chen, Aaron Trigg, Tej Poudel Chhetri, David P. Young, Igor Dubenko, Naushad Ali, and Shane Stadler 
Louisiana State University

LSU Digital Commons

$6-7-2020$

\section{The influence of Au substitution and hydrostatic pressure on the phase transitions and magnetocaloric properties of $\mathrm{MnCoGe}$ alloys}

Jing Han Chen

Louisiana State University

Aaron Trigg

Louisiana State University

Tej Poudel Chhetri

Louisiana State University

David P. Young

Louisiana State University

Igor Dubenko

Southern Illinois University Carbondale

See next page for additional authors

Follow this and additional works at: https://digitalcommons.Isu.edu/physics_astronomy_pubs

\section{Recommended Citation}

Chen, J., Trigg, A., Poudel Chhetri, T., Young, D., Dubenko, I., Ali, N., \& Stadler, S. (2020). The influence of Au substitution and hydrostatic pressure on the phase transitions and magnetocaloric properties of MnCoGe alloys. Journal of Applied Physics, 127 (21) https://doi.org/10.1063/5.0007172 


\section{Authors}

Jing Han Chen, Aaron Trigg, Tej Poudel Chhetri, David P. Young, Igor Dubenko, Naushad Ali, and Shane Stadler 


\section{The influence of Au substitution and}

hydrostatic pressure on the phase transitions and magnetocaloric properties of MnCoGe alloys

Cite as: J. Appl. Phys. 127, 213901 (2020); https://doi.org/10.1063/5.0007172

Submitted: 09 March 2020 - Accepted: 15 May 2020 • Published Online: 01 June 2020

Jing-Han Chen, Aaron Trigg, Tej Poudel Chhetri, et al.
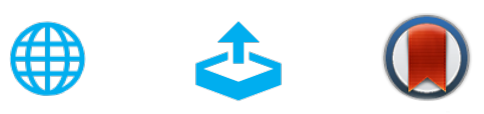

\section{ARTICLES YOU MAY BE INTERESTED IN}

Giant reversible barocaloric response of $(\mathrm{MnNiSi})_{1-x}\left(\mathrm{FeCoGe}_{\mathrm{X}}(\mathrm{x}=0.39,0.40,0.41)\right.$

APL Materials 7, 061106 (2019); https://doi.org/10.1063/1.5097959

Giant magnetocaloric effects by tailoring the phase transitions

Applied Physics Letters 96, 172504 (2010); https://doi.org/10.1063/1.3399773

Fabrication of magnetocaloric $\mathrm{La}(\mathrm{Fe}, \mathrm{Si})_{13}$ thick films

Journal of Applied Physics 127, 215103 (2020); https://doi.org/10.1063/5.0006324

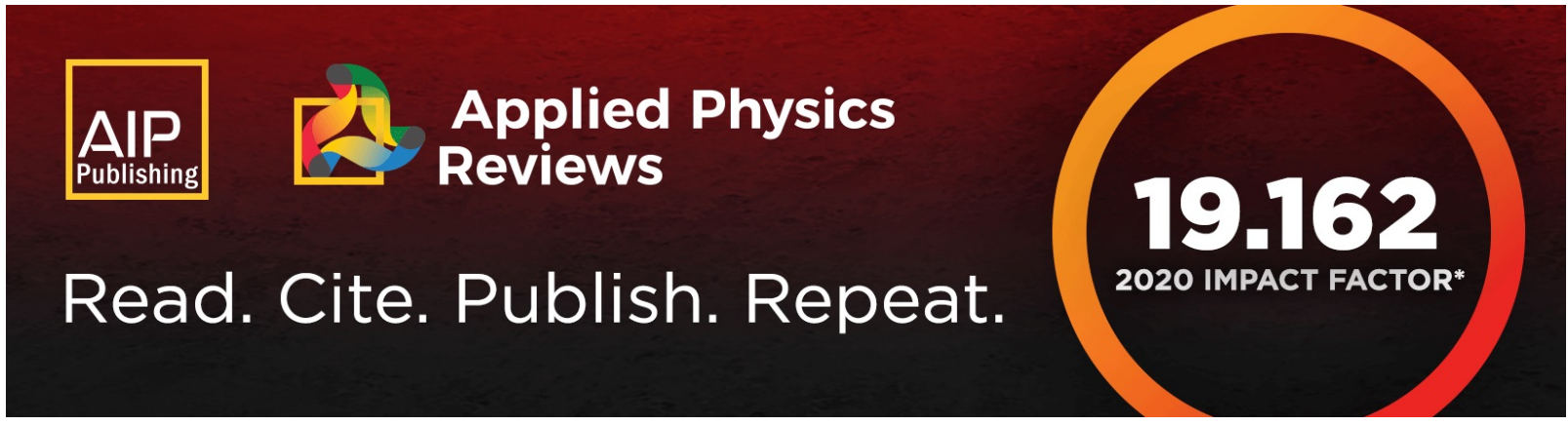




\title{
The influence of Au substitution and hydrostatic pressure on the phase transitions and magnetocaloric properties of MnCoGe alloys
}

Cite as: J. Appl. Phys. 127, 213901 (2020); doi: 10.1063/5.00071 72

Submitted: 9 March 2020 - Accepted: 15 May 2020 .

Published Online: 1 June 2020

Jing-Han Chen, ${ }^{1, a)}$ Aaron Trigg, ${ }^{7}$ Tej Poudel Chhetri, ${ }^{1}$ David P. Young, ${ }^{7}$ Igor Dubenko, ${ }^{2}$ Naushad Ali, ${ }^{2}$ and Shane Stadler ${ }^{\top}$

\author{
AFFILIATIONS \\ ${ }^{7}$ Department of Physics and Astronomy, Louisiana State University, Baton Rouge, Louisiana 70803, USA \\ ${ }^{2}$ Department of Physics, Southern Illinois University, Carbondale, Illinois 62901, USA
}

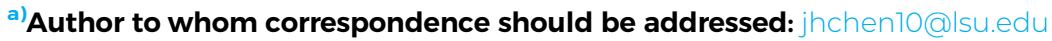

\begin{abstract}
In this work, the phase transitions and magnetocaloric properties of $\mathrm{Mn}_{1-x} \mathrm{Au}_{x} \mathrm{CoGe}(0 \leq x \leq 0.025)$ alloys were studied as a function of concentration $x$ and applied hydrostatic pressure. The increasing substitution of $\mathrm{Au}$ for $\mathrm{Mn}$ results in the decrease of the first-order martensitic transition temperature, and this first-order martensitic transition was ultimately converted to a second-order magnetic transition when the Au substitution $(x)$ reached 0.025 . The magnitudes of the maximum magnetic entropy changes increased when the magnetic and structural transitions were coupled, which occurred for $0.005 \leq x \leq 0.020$. The largest maximum magnetic entropy change for a field change of $\mu_{0} \Delta H=7 \mathrm{~T}$ was $33.1 \mathrm{~J} / \mathrm{kg} \mathrm{K}$ for the sample with $x=0.020$. Similar to the effect of Au substitution, the first-order martensitic transition temperature initially decreased, and then converted to second order, when the applied hydrostatic pressure reached a large enough value. Interestingly, both Au substitution and pressure application cause a volume reduction and, in both cases, the first-order martensitic transition temperature initially reduced and then converted to second-order. These results suggest two different methods of tuning the transition temperatures in these magnetocaloric materials. One can either apply hydrostatic pressure and temporarily adjust the transition temperatures or modify the composition chemically and permanently change the transition temperatures.
\end{abstract}

Published under license by AIP Publishing. https://doi.org/10.1063/5.0007172

\section{INTRODUCTION}

Due to the desire to replace conventional vapor compression systems, and the predicted high efficiency of magnetic refrigeration, the study of the magnetocaloric effect (MCE) has intensified. ${ }^{1-7}$ The increasing effort to discover better magnetocaloric materials has persisted since a giant $\mathrm{MCE}$ in $\mathrm{Gd}_{5}(\mathrm{Si}, \mathrm{Ge})_{4}$ with a first-order phase transition near room temperature was discovered. ${ }^{8}$ Since then, many types of materials have been discovered and proposed as candidates for magnetic refrigeration working materials, including the Ni-Mn based Heusler alloys, ${ }^{9-17}$ MnAs-based compounds, ${ }^{18-20}$ $\mathrm{LaFe}_{13-x} \mathrm{Si}_{x}$ related compounds, ${ }^{21,22}$ and intermetallic MnTX ( $T=$ transition metal and $X=\mathrm{Ge}, \mathrm{Si}$ ) systems. ${ }^{23-34}$

Among the above-mentioned material candidates, intermetallic MnTX systems have drawn increasing attention recently due to their giant MCEs caused by large volume expansion/contraction resulting from first-order magneto-structural transitions between a $\mathrm{Ni}_{2}$ In-type hexagonal structure (space group: $P 6_{3} / m m c$ ) and a TiNiSi-type orthorhombic structure (space group: Pnma). ${ }^{29-31}$ Considerable interest has been drawn particularly to the MnCoGe-based and MnNiGe-based systems since their martensitic transitions located relatively close to room temperature can be tuned by stoichiometry modification, ${ }^{35-37}$ doping with interstitial atoms, ${ }^{38}$ vacancy tuning, ${ }^{39,40}$ or pressure application. ${ }^{28}$ In equiatomic $\mathrm{MnCoGe}$, a first-order martensitic transition occurs from a paramagnetic high temperature hexagonal $\mathrm{Ni}_{2}$ In-type phase to a paramagnetic low-temperature orthorhombic TiNiSi-type phase with a transition at $470 \mathrm{~K}$, and is ordered ferromagnetically below its Curie temperature, $T_{C}=345 \mathrm{~K}^{41,42}$ It has been recently shown that the Curie temperatures and martensitic transition temperatures of MnCoGe compounds can be controlled by partially substituting 
for $\mathrm{Mn}$ atoms with $\mathrm{Ag},{ }^{43} \mathrm{Al},{ }^{44} \mathrm{Cr},{ }^{45,46} \mathrm{Cu},{ }^{47,48} \mathrm{Fe},{ }^{49-52} \mathrm{Ni}^{53}$ or $\mathrm{V},{ }^{54}$ or for Co atoms with $\mathrm{Al},{ }^{55} \mathrm{Cd},{ }^{56} \mathrm{Cu},{ }^{48} \mathrm{Fe},{ }^{42,57,58}$ or $\mathrm{Zn},{ }^{59,60}$ or for Ge atoms with $\mathrm{Ga},{ }^{61} \mathrm{In},{ }^{62}$ or $\mathrm{Sn} .{ }^{34}$

In this work, we report the effects of Au-substitution and applied hydrostatic pressure on the structural and magnetic transitions in $\mathrm{Mn}_{1-x} \mathrm{Au}_{x} \mathrm{CoGe}(0 \leq x \leq 0.025)$. We have found that, as the Au concentration $(x)$ increases, the first-order martensitic transition temperature shifts from above room temperature $(442 \mathrm{~K})$ to $263 \mathrm{~K}$, and then completely converts to a second order phase transition for the sample with $x=0.025$. For the samples with $0.005 \leq x \leq 0.020$, the magnitudes of the maximum magnetic entropy changes were enhanced since the magnetic and structural transitions are coupled. The largest maximum magnetic entropy change for a field change of $\mu_{0} \Delta H=7 \mathrm{~T}$ was found to be $33.1 \mathrm{~J} /$ $\mathrm{kg} \mathrm{K}$ for the sample with $x=0.020$. In addition, room temperature $\mathrm{x}$-ray diffraction shows a volume reduction as the Au-substitution increases.

Similar to the effect of Au substitution, the first-order martensitic transition temperature decreases initially and then converts to second order as the applied hydrostatic pressure increases. It is worth noting that both $\mathrm{Au}$ substitution and pressure application effectively cause a volume reduction and, in both cases, the firstorder martensitic transition temperature decreases initially and then converts to second order.

\section{SAMPLE PREPARATION}

$\mathrm{Mn}_{1-x} \mathrm{Au}_{x} \mathrm{CoGe}(x=0.000,0.005,0.010,0.015,0.020$, and $0.025)$ alloys were prepared from high purity $\mathrm{Mn}$ (99.95\%), Co (99.95\%), Ge (99.999\%), and $\mathrm{Au}$ (99.99\%) elemental components by sealing the raw elements with the desired compositions in a quartz tube under vacuum and then heat treating at $1100{ }^{\circ} \mathrm{C}$ for $12 \mathrm{~h}$ in a tube furnace. To ensure the homogeneity of the chemical compositions, the ingots were flipped and heat treated at $1100^{\circ}$ for another $12 \mathrm{~h}$. The sample labels and their corresponding thermomagnetic properties are summarized in Table I.

\section{THERMAL ANALYSIS}

To explore the formation of these MnCoGe-type compounds, simultaneous differential scanning calorimetry (DSC) measurements and thermogravimetric analysis (model: SDT Q600 manufactured by TA Instruments, Inc.) were employed. The heat flow curves of the parent compound $\mathrm{MnCoGe}$ as a function of temperature measured up to $1100{ }^{\circ} \mathrm{C}$ are shown in Fig. 1. The first-order phase transition with thermal hysteresis due to the melting and crystallization of the MnCoGe ingot was observed between $1025^{\circ} \mathrm{C}$ and $1075^{\circ} \mathrm{C}$. The first-order martensitic transition was detected between $100^{\circ} \mathrm{C}$ and $250^{\circ} \mathrm{C}$ (see the inset of Fig. 1). No other perceivable first-order phase transitions were observed. Taking this thermal analysis as a guideline, all of the $\mathrm{Mn}_{1-x} \mathrm{Au}_{x} \mathrm{CoGe}$ samples in this work were prepared by melting at $1100^{\circ} \mathrm{C}$ twice to ensure a thorough and homogeneous synthesis.

\section{X-RAY DIFFRACTION}

To investigate the crystal structures of the samples, powder $\mathrm{x}$-ray diffraction (XRD) measurements were performed using a
TABLE I. The compositions of the $\mathrm{Mn}_{1-x} \mathrm{Au}_{x} \mathrm{CoGe}$ samples and their corresponding thermomagnetic properties.

\begin{tabular}{ccccccc}
\hline \hline Label & $x(\%)$ & $\begin{array}{c}T_{m}{ }^{\mathrm{a}} \\
(\mathrm{K})\end{array}$ & $\begin{array}{c}-\Delta S_{\max }{ }^{\mathrm{b}} \\
(\mathrm{J} / \mathrm{kg} \mathrm{K})\end{array}$ & $\begin{array}{c}\delta_{F W H M}{ }^{\mathrm{c}} \\
(\mathrm{K})\end{array}$ & $\begin{array}{c}-R C P^{\mathrm{d}} \\
(\mathrm{J} / \mathrm{kg})\end{array}$ & $\begin{array}{c}M_{\text {sat }} / f . \\
u .\left(\mu_{B}\right)\end{array}$ \\
\hline Au00 & 0.0 & 442 & $\ldots$ & $\ldots$ & $\ldots$ & 4.07 \\
Au05 & 0.5 & 355 & 13.4 & 30.4 & 407 & 3.87 \\
Au10 & 1.0 & 304 & 29.9 & 15.7 & 469 & 3.87 \\
Au15 & 1.5 & 276 & 33.1 & 12.0 & 397 & 3.96 \\
Au20 & 2.0 & 263 & 30.4 & 11.4 & 347 & 4.06 \\
Au25 & 2.5 & $\ldots$ & $\ldots$ & $\ldots$ & $\ldots$ & 3.20 \\
\hline \hline
\end{tabular}

${ }^{\mathrm{a}} T_{m}$ is the martensitic transition temperature estimated from temperature-dependent isofield magnetization measurements, except $T_{m}$ for sample Au00 which was estimated from the calorimetric measurement.

${ }^{\mathrm{b}} \Delta S_{\max }$ is the maximum magnetic entropy change for a field change of $\mu_{0} \Delta H=7 \mathrm{~T}$ calculated from isothermal magnetization measurements described in Sec. VI.

${ }^{\mathrm{c}} \delta_{F W H M}$ is the full width at half maximum of the entropy $\Delta S(T)$.

${ }^{\mathrm{d}} R C P$ is the relative cooling power, estimated as $R C P=\Delta S_{\max } \times \delta_{F W H M}$, for $\mu_{0} \Delta H=7 \mathrm{~T}^{63,64}$

Scintag XDS2000 powder diffractometer with $\mathrm{Cu} \mathrm{K} \alpha$ radiation at room temperature. Since these materials are sensitive to pressure, ${ }^{65-67}$ instead of grinding the samples, the powder samples used in XRD measurements were prepared by temperature cycling the samples through their transitions until they spontaneously broke into powders. Figure 2 shows the XRD results for all samples at room temperature.

Figure 2 shows that sample Au00 stabilizes in the TiNiSi-type orthorhombic structure at room temperature, and that the fraction

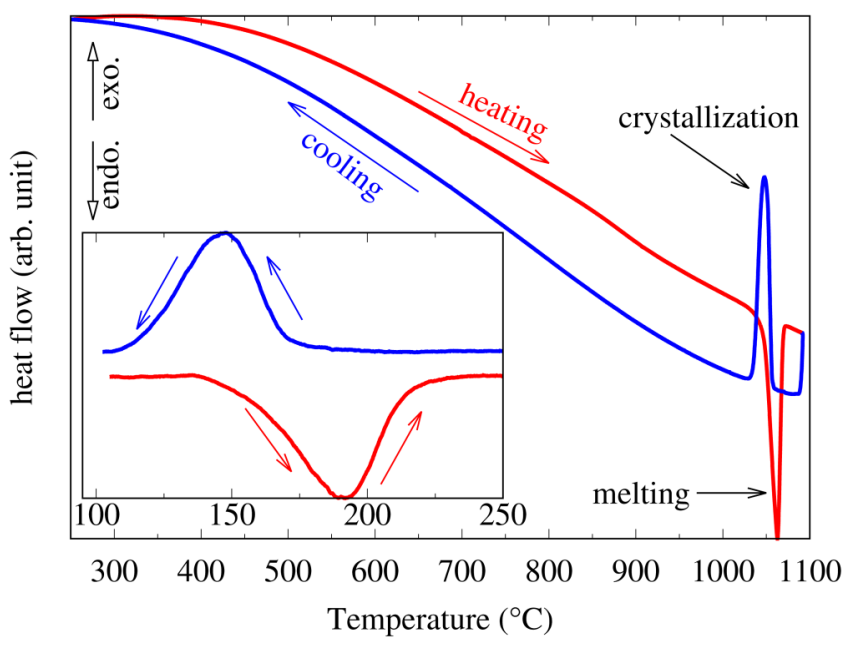

FIG. 1. The heat flow data of a MnCoGe ingot obtained from DSC measurements. The ingot was melted completely at $1100^{\circ} \mathrm{C}$. Except for the first-order martensitic transition shown in the inset, and the melting/crystallization point, no other perceivable first-order phase transitions were observed. The experimental heat directions are indicated by the arrows. 


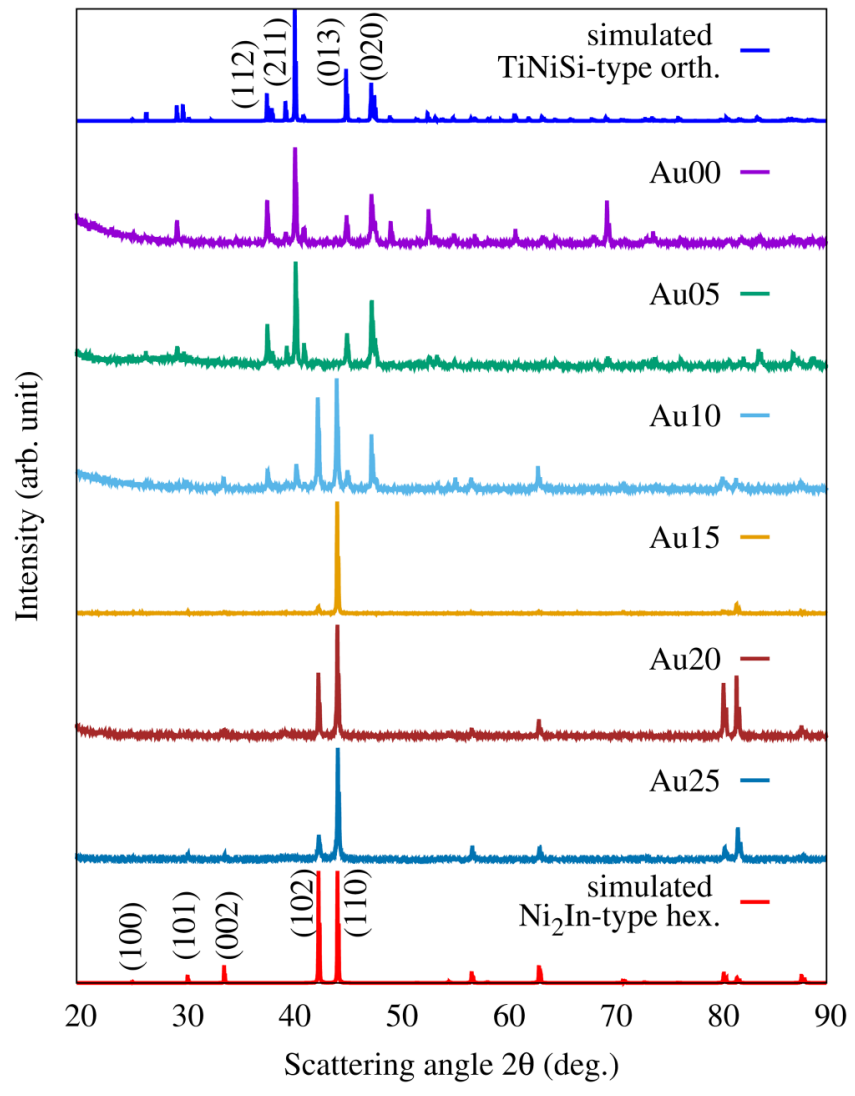

FIG. 2. Cu K $\alpha$ powder $\mathrm{x}$-ray diffraction patterns of all samples at room temperature. The top panel shows the simulated TiNiSi-type orthorhombic pattern, and the bottom panel shows the simulated $\mathrm{Ni}_{2}$ In-type hexagonal pattern.

of the $\mathrm{Ni}_{2}$ In-type hexagonal structure increases with increasing $\mathrm{Au}$ substitution since the first order martensitic transition temperature decreases through room temperature. As the $\mathrm{Au}$ substitution increased to $2.5 \%$ (i.e., sample Au25), a trace secondary phase was detected at room temperature that could be either an untransformed portion of the TiNiSi-type martensite phase and/or an undetermined crystalline phase. The lattice constants and volumes from the Rietveld refinements using General Structure Analysis System (GSAS) software ${ }^{68-70}$ are tabulated in Table II. The total

TABLE II. The lattice constants and cell volumes obtained from XRD refinements of powder diffraction data collected at room temperature.

\begin{tabular}{lccccc}
\hline \hline Label & Major phase & $a(\AA)$ & $b(\AA)$ & $c(\AA)$ & vol./f.u. $\left(\AA^{3}\right)$ \\
\hline Au00 & TiNiSi & 5.963 & 3.822 & 7.060 & 40.23 \\
Au05 & TiNiSi & 5.949 & 3.823 & 7.055 & 40.11 \\
Au10 & $\mathrm{Ni}_{2} \mathrm{In}$ & 4.087 & 4.087 & 5.312 & 38.42 \\
Au15 & $\mathrm{Ni}_{2} \mathrm{In}$ & 4.084 & 4.084 & 5.307 & 38.34 \\
Au20 & $\mathrm{Ni}_{2} \mathrm{In}$ & 4.082 & 4.082 & 5.305 & 38.28 \\
Au25 & $\mathrm{Ni}_{2} \mathrm{In}$ & 4.080 & 4.080 & 5.303 & 38.23 \\
\hline \hline
\end{tabular}

volumes per formula unit and lattice constants of the major phase at room temperature decrease as the $\mathrm{Au}$ concentration increases. Meanwhile, as will be shown explicitly in Sec. V, the martensitic transition temperatures relative to the parent $\mathrm{MnCoGe}$ alloy (i.e., sample Au00) decreases with increasing Au substitution until the $\mathrm{Au}$ substitution reached 2.5\% (i.e., sample Au25) when the firstorder martensitic transition was completely suppressed.

It is known that the TiNiSi-type structure can be regarded as an orthorhombic distortion of the $\mathrm{Ni}_{2}$ In-type hexagonal structure. $^{67}$ The lattice parameters of the two structures are related through $a_{\text {orth }}=c_{\text {hex }}, b_{\text {orth }}=a_{\text {hex }}$, and $c_{\text {orth }}=\sqrt{3} a_{\text {hex }}$. The composition dependences of the unit cell parameters calculated from the room temperature XRD measurements are shown in Fig. 3. As the $\mathrm{Au}$ substitution increases, the volume of the TiNiSi-type orthorhombic martensite phase decreases, resulting from the effect of a reduced $a_{o r t h}$ and expanded $b_{\text {orth }}$, and then the $\mathrm{Ni}_{2}$ In-type hexagonal austenite phase which has smaller volume becomes more stable. As the Au substitution $(x)$ reached 0.025 , the volume of the sample reduced by $4.2 \%$ and the structural transition from the $\mathrm{Ni}_{2} \mathrm{In}$-type hexagonal to the TiNiSi-type orthorhombic becomes completely

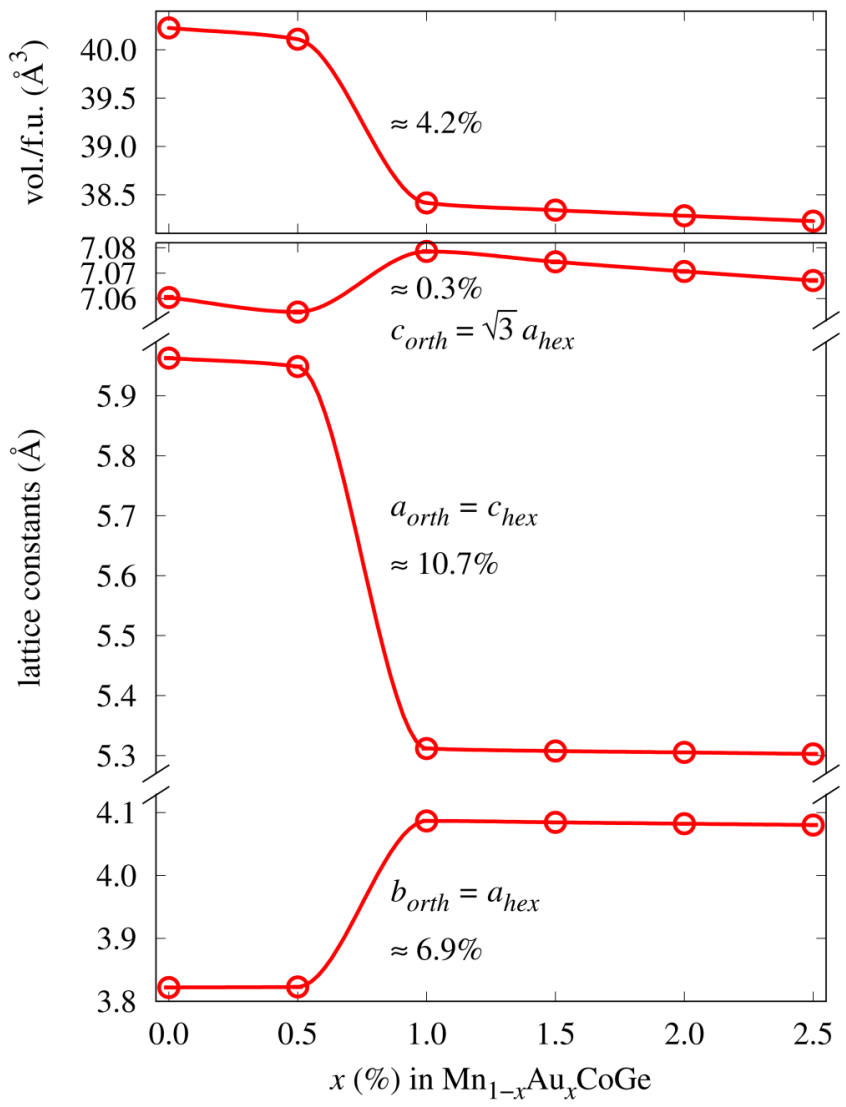

FIG. 3. Lattice constants and cell volumes vs substitution percentage $x$. The distortion due to a reduced $a_{\text {orth }}$ and an expanded $b_{\text {orth }}$ was observed, while $c_{\text {orth }}$ remains nearly constant. 
absent. It is worth noting that the deformations of the cell parameters $a_{\text {orth }}=c_{\text {hex }}$ and $b_{\text {orth }}=a_{\text {hex }}$ are relatively larger than that of $c_{\text {orth }}=\sqrt{3} a_{\text {hex }}$, which is commonly observed in these alloy systems with a structural transition from a $\mathrm{Ni}_{2} \mathrm{In}$-type hexagonal structure to a TiNiSi-type orthorhombic structure. ${ }^{66,67}$

\section{MAGNETIZATION MEASUREMENTS AND ANALYSIS}

The magnetization measurements were performed using a Magnetic Property Measurement System manufactured by Quantum Design operating within the temperature range $10-400 \mathrm{~K}$, and in applied magnetic fields up to $7 \mathrm{~T}$. The isofield, temperaturedependent magnetization measurements were performed using fieldcooled cooling and field-cooled warming protocols, and the results are shown in Fig. 4. First-order martensitic transitions with thermal hysteresis were observed (for samples Au05, Au10, Au15, and

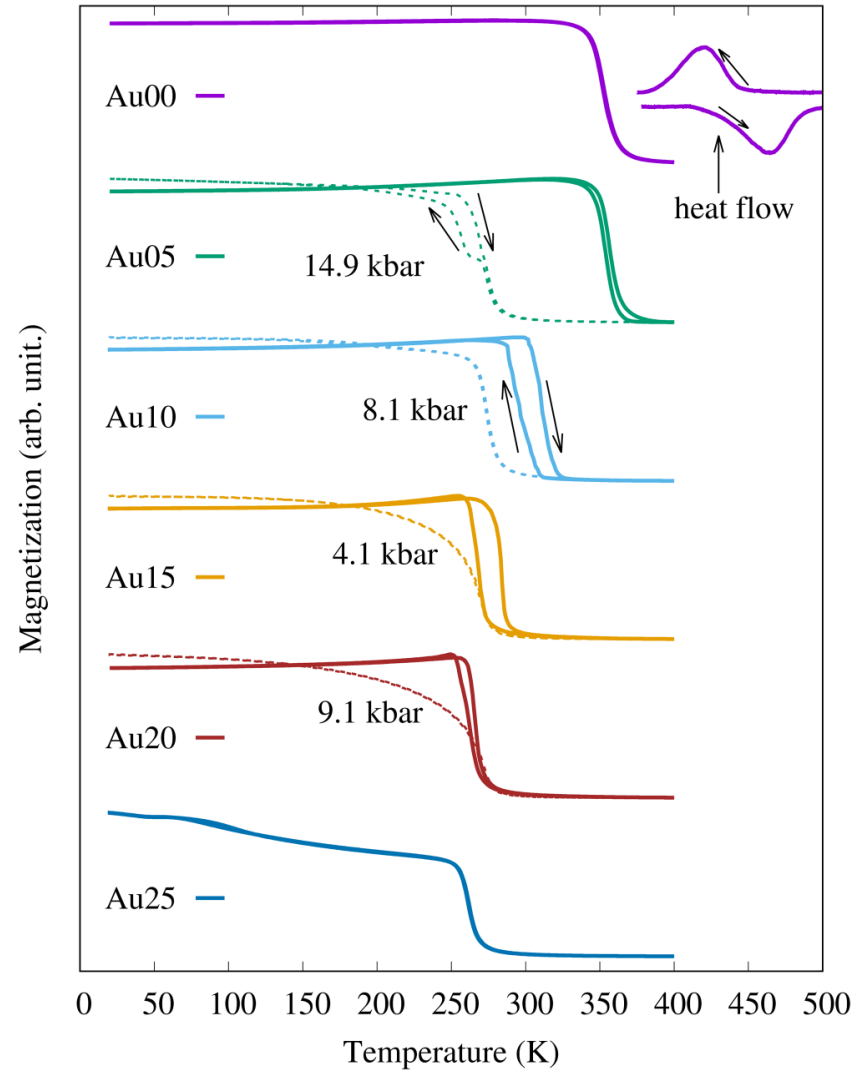

FIG. 4. The temperature-dependent magnetization of all samples in an applied field of $\mu_{0} \mathrm{H}=0.1 \mathrm{~T}$ (solid lines represent data taken at atmospheric pressure, whereas dashed lines represent data taken at the indicated applied pressures) measured using field-cooled cooling and field-cooled warming protocols. The arrows shown for sample Au10 (solid lines) and sample Au05 (dashed lines, $14.9 \mathrm{kbar}$ ) represent the temperature sweep directions of measurements. For sample Au00, a DSC measurement above $400 \mathrm{~K}$ (labeled as "heat flow" in the figure) was performed and the experimental directions of temperature changes are indicated by arrows.
Au20), and the transition temperatures decreased as the Au substitution increased up to $2.5 \%$. The transition temperatures were estimated from the isofield magnetization measurements at $0.1 \mathrm{~T}$ and are listed in Table I.

Figure 5 shows the magnetic and structural phase diagram of $\mathrm{Mn}_{1-x} \mathrm{Au}_{x} \mathrm{CoGe}$ for $0 \% \leq x \leq 2.5 \%$. For compositions between $x=0.5 \%$ and $2 \%$, first-order magneto-structural transitions wereobserved, and a ferromagnetic (FM) TiNiSi-type orthorhombic structure directly transformed to a paramagnetic (PM) $\mathrm{Ni}_{2}$ In-type hexagonal structure without going through a second-order magnetic transition which often results in large field induced magnetic entropy changes. The effect of reducing the martensitic transition in $\mathrm{MnCoGe}$ alloys via partial substitution for $\mathrm{Mn}$ atoms was also observed previously by utilizing different elements, such as $\mathrm{Al}^{44}$ $\mathrm{Cr},{ }^{45,46} \mathrm{Cu},{ }^{47,48} \mathrm{Fe},{ }^{49-52} \mathrm{Ni}^{53}$ or $\mathrm{V} .{ }^{54}$ Note that these elements, as well as Au used in the current work, have atomic radii not larger than the $\mathrm{Mn}$ atoms they are replacing. ${ }^{71}$ However, in the case where Ag, which has a radius that is larger ${ }^{71}$ than $\mathrm{Mn}$, was used for the substitution, ${ }^{43}$ the martensitic transition temperatures remain above $400 \mathrm{~K}$ and the magnetic second-order phase transition of the TiNiSi-type orthorhombic structure moved to higher temperatures.

Isothermal magnetization measurements at $T=2.0 \mathrm{~K}$ were performed in order to investigate the saturation magnetization, the results of which are shown in Fig. 6 . The saturation magnetization $\left(M_{\text {sat }}\right)$ values in Table I were estimated by fitting the experimental magnetization data in high magnetic field using the law of approach-to-saturation

$$
M=M_{\text {sat }}\left(1-\frac{a}{H^{2}}-\frac{b}{H^{3}}\right)
$$

where $a$ and $b$ are fitting parameters. ${ }^{72,73}$ Without the application of hydrostatic pressure, all of the samples showing martensitic

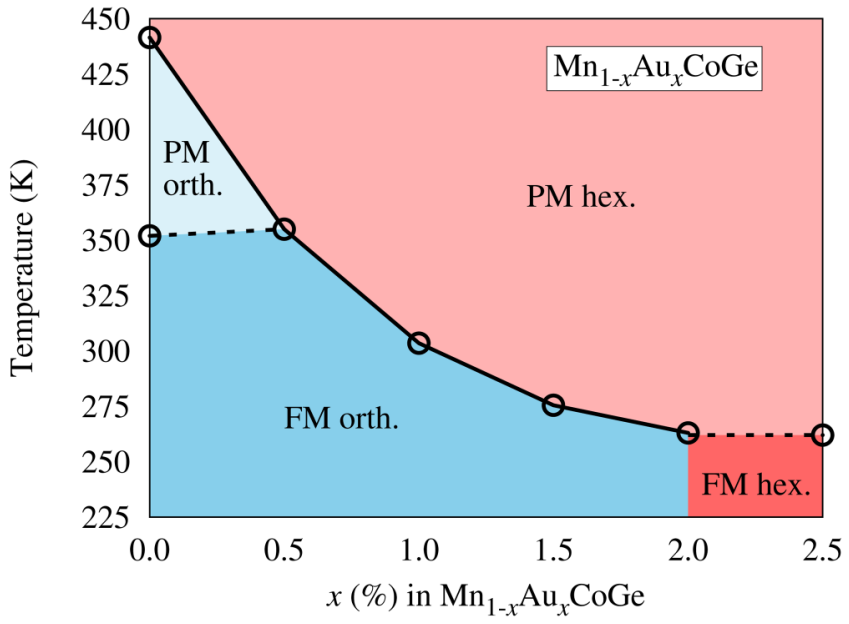

FIG. 5. The magnetic and structural phase diagram of $M_{1-x} A u_{x} \mathrm{CoGe}$ were constructed using the experimental results in the current work. For $0.5 \% \leq x \leq 2.0 \%$, the structural and magnetic transition are coupled. 


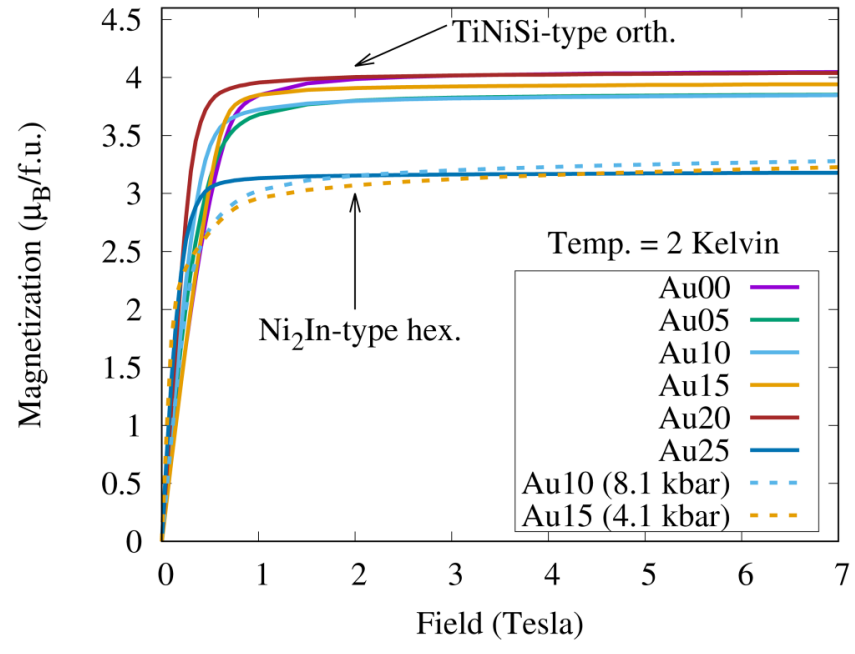

FIG. 6. The isothermal magnetization data of all samples at $T=2.0 \mathrm{~K}$. The lower saturation magnetic moments of samples Au25 (without pressure), Au10 (8.1 kbar), and Au15 (4.1 kbar) were observed because the low temperature TiNiSi-type orthorhombic structure was suppressed and hence these data reflect the lower magnetic moments of the $\mathrm{Ni}_{2}$ In-type hexagonal structure.

transitions stabilized in the TiNiSi-type orthorhombic crystal structure at $2.0 \mathrm{~K}$ so that their magnetic saturation values are nearly the same, as shown in Fig. 6, while the measured saturation magnetization of sample Au25, for which the martensitic transition is absent, turns out to be lower than those of the others. These saturation magnetization results are consistent with other reports, ${ }^{74,75}$ in which the magnetic moments of the MnCoGe parent phase are found to be 3.9 and $2.6 \mu_{B} /$ f.u. for the TiNiSi-type orthorhombic and $\mathrm{Ni}_{2} \mathrm{In}$-type hexagonal structures, respectively.

Magnetic measurements under hydrostatic pressure were performed using a $\mathrm{BeCu}$ cylindrical pressure cell manufactured by HMD, Japan. Daphne 7373 oil was used as the pressure transmitting medium. The magnitude of the applied pressure was calibrated by measuring the shift in the superconducting transition temperature of $\mathrm{Sn}$, which was placed in the cell with the sample as a reference manometer. The isofield magnetization results in Fig. 4 (dashed lines) demonstrate the effects of pressure on the martensitic transitions for sample Au05 (14.9 kbar), Au10 (8.1 kbar), Au15 (4.1 kbar), and Au20 (9.1 kbar) under the specified hydrostatic pressures. With the specified hydrostatic pressures, the first-order martensitic transitions of sample Au10, Au15, and Au20 were completely converted to second-order magnetic transitions while, for sample Au05 under $14.9 \mathrm{kbar}$, the first-order martensitic transition remained but the transition temperature was reduced by over $150 \mathrm{~K}$, corresponding to a rate of $10 \mathrm{~K} / \mathrm{kbar}$, which is close to what has been found previously in $\mathrm{MnCoGe}^{76}$ and suggests that large barocaloric effects might be found in these systems. It is reasonable to expect the disappearance of the first-order martensitic transition in Au05 if the applied pressure is large enough. However, the applied pressure in sample Au05 (14.9 kbar) is close to the upper limit of attainable hydrostatic pressures for the cell that was used. Generally speaking, the martensitic transition temperatures were reduced with the increase of applied pressure, or $\mathrm{Au}$ substitution, until they were completely converted to second-order magnetic transitions and the thermal hysteresis disappeared. It is also worth noting that the low values of the saturation magnetic moments of samples Aul0 (8.1 kbar) and Au15 $(4.1 \mathrm{kbar})$ at $2.0 \mathrm{~K}$ shown in Fig. 6 were observed since the low temperature TiNiSi-type orthorhombic structure was suppressed under the specified pressures, and the magnetic moments of $\mathrm{Ni}_{2}$ In-type hexagonal structure are lower than that of the TiNiSi-type orthorhombic structure.

\section{MAGNETIC-INDUCED ISOTHERMAL ENTROPY}

The performance of MCE materials can be quantified by the magnetic entropy change in an isothermal process. ${ }^{77}$ Experimentally, the magnetic entropy change is estimated from isothermal magnetization data using the thermodynamic relation

$$
\begin{aligned}
& \Delta S(T, 0 \rightarrow H)=\frac{\partial}{\partial T}\left(\int_{0}^{H} M\left(T, H^{\prime}\right) d H^{\prime}\right)_{T=\text { const. }} \\
& \quad \cong \frac{1}{\Delta T}\left[\int_{0}^{H} M\left(T+\Delta T, H^{\prime}\right) d H^{\prime}-\int_{0}^{H} M\left(T, H^{\prime}\right) d H^{\prime}\right] .
\end{aligned}
$$

Therefore, a series of isothermal magnetization experiments were performed to obtain $M(T=$ const., $H)$. In order to eliminate a potential residual ferromagnetic martensite phase generated in each isothermal magnetization measurement, which may result in inaccurate entropy changes, the samples were heated to complete the paramagnetic austenite phase, and then cooled down to the target measurement temperature under zero field before initiating each isothermal magnetization measurement. The magnetic entropy

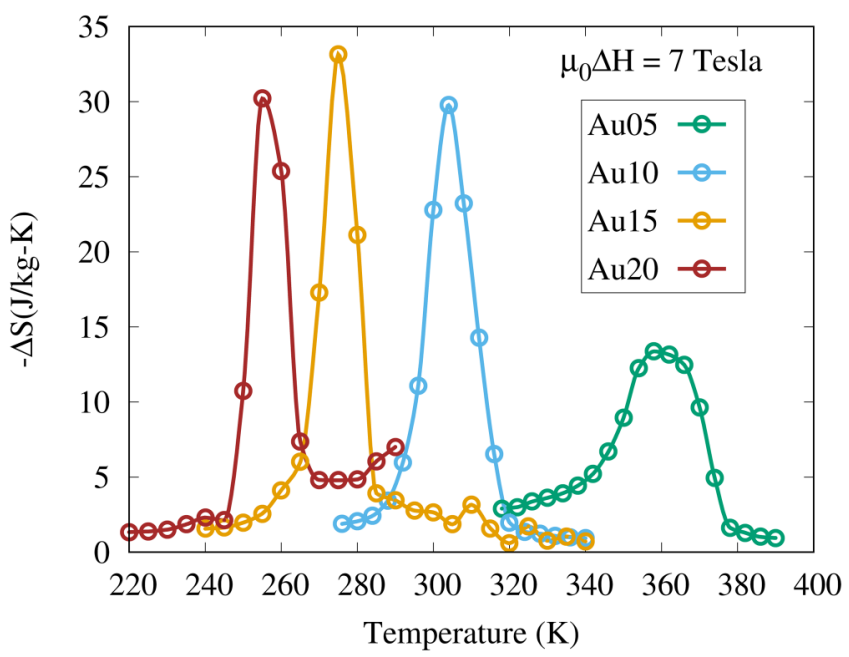

FIG. 7. The magnetic entropy changes for $\mu_{0} \Delta H=7 \mathrm{~T}$ at the corresponding first-order magneto-structural transitions under ambient pressure were estimated from magnetization isotherm measurements. 
change determined using this thermodynamic reset protocol should represent the magnetic entropy change in the direction from the austenite phase to the martensite phase. This protocol was referred to as the cooling protocol, or the loop process, in Refs. 78 and 79, because the results obtained using this protocol can also be acquired by calorimetric experiments during the cooling process.

The magnetic entropy changes for $\mu_{0} \Delta H=7 \mathrm{~T}$ as a function of temperature are shown in Fig. 7. The values of the maximum magnetic entropy changes $\left(\Delta S_{\max }\right)$ for the samples showing firstorder magneto-structural transitions are listed in Table I. It is interesting that samples Au10, Au15, and Au20 show comparable magnitudes of maximum magnetic entropy changes and the full width at half maximum of the entropy, as shown in Table I, while sample Au05 shows the smallest maximum magnetic entropy change and the largest $\delta_{F W H M}\left(\Delta S_{\max }=-13.4 \mathrm{~J} / \mathrm{kg} \mathrm{K}, \delta_{F W H M}=30.4 \mathrm{~K}\right)$. In addition, the largest relative cooling power ${ }^{80}$ was observed in sample Au10 $(R C P=-469 \mathrm{~J} / \mathrm{kg})$, while the smallest relative cooling power was observed in sample Au20 $(R C P=-347 \mathrm{~J} / \mathrm{kg})$. These magnitudes of $\Delta S_{\max }$ and $R C P$ reported here are comparable with previously reported $\mathrm{MnNiSi}$-type magnetocaloric materials that undergo the same magneto-structural transitions from a ferromagnetic TiNiSi-type orthorhombic structure to a paramagnetic $\mathrm{Ni}_{2}$ In-type hexagonal structure. ${ }^{6,67}$

\section{CONCLUSIONS}

In conclusion, the structural and magnetic properties of $\mathrm{Mn}_{1-x} \mathrm{Au}_{x} \mathrm{CoGe}(x=0.000,0.005,0.010,0.015,0.020$, and 0.025$)$ under hydrostatic pressures were investigated. First-order martensitic transitions from a $\mathrm{Ni}_{2}$ In-type hexagonal austenite phase to a TiNiSi-type orthorhombic martensite phase were observed, and the martensitic transition temperatures decreased by up to $180 \mathrm{~K}$ via either $\mathrm{Au}$ substitution or hydrostatic pressure application. As the substitution percentage $(x)$ reached $2.5 \%$, the first-order martensitic transition was suppressed, and only a second-order magnetic phase transition was observed. Similarly, as the hydrostatic pressure increased, the first-order martensitic transition temperature initially decreased, and then converted to a $\mathrm{Ni}_{2} \mathrm{In}$-type hexagonal structure with only second-order phase transitions at high pressures. It is worth noting that, in this work, the reduction of the martensitic transition temperatures was found to be a consequence of the volume reduction, which can occur either permanently by modifying the chemical composition or temporarily by applying hydrostatic pressure.

\section{ACKNOWLEDGMENTS}

S. Stadler and J.-H. Chen acknowledge support from the U.S. Department of Energy, Office of Basic Energy Sciences under Award No. DE-FG02-13ER46946. N. Ali and I. Dubenko acknowledge support from the U.S. Department of Energy, Office of Basic Energy Sciences under Award No. DE-FG02-06ER46291. D. P. Young acknowledges support from the U.S. National Science Foundation, Division of Materials Research under Award No. NSF-DMR-1904636. The DSC data used in this publication were collected at the Center for Advanced Microstructures and Devices (CAMD) under the supervision of A. Roy.

\section{DATA AVAILABILITY}

The data that support the findings of this study are available from the corresponding author upon reasonable request.

\section{REFERENCES}

${ }^{1}$ E. Brück, O. Tegus, D. C. Thanh, N. T. Trung, and K. H. J. Buschow, Int. J. Refrig. 31, 763 (2008).

${ }^{2}$ K. G. Sandeman, Scr. Mater. 67, 566 (2012).

${ }^{3}$ E. Brück, J. Phys. D 38, R381 (2005).

${ }^{4}$ B. G. Shen, J. R. Sun, F. X. Hu, H. W. Zhang, and Z. H. Cheng, Adv. Mater. 21, 4545 (2009).

${ }^{\mathbf{5}}$ K. A. Gschneidner and V. K. Pecharsky, Int. J. Refrig. 31, 945 (2008).

${ }^{6}$ K. A. Gschneidner, Jr., V. K. Pecharsky, and A. O. Tsokol, Rep. Prog. Phys. 68, 1479 (2005).

${ }^{7}$ V. Franco, J. S. Blázquez, B. Ingale, and A. Conde, Annu. Rev. Mater. Res. 42, 305 (2012)

${ }^{8}$ V. K. Pecharsky and K. A. Gschneidner, Jr., Phys. Rev. Lett. 78, 4494 (1997).

${ }^{9}$ Y. Sutou, Y. Imano, N. Koeda, T. Omori, R. Kainuma, K. Ishida, and K. Oikawa, Appl. Phys. Lett. 85, 4358 (2004).

${ }^{10}$ T. Krenke, E. Duman, M. Acet, E. F. Wassermann, X. Moya, L. Mañosa, and A. Planes, Nat. Mater. 4, 450 (2005).

${ }^{11}$ K. Oikawa, W. Ito, Y. Imano, Y. Sutou, R. Kainuma, K. Ishida, S. Okamoto, O. Kitakami, and T. Kanomata, Appl. Phys. Lett. 88, 122507 (2006).

${ }^{12}$ M. Khan, N. Ali, and S. Stadler, J. Appl. Phys. 101, 053919 (2007).

${ }^{13}$ F. S. Liu, Q. B. Wang, W. Q. Ao, Y. J. Yu, L. C. Pan, and J. Q. Li, J. Magn. Magn. Mater. 324, 514 (2012).

${ }^{14}$ L. H. Bennett, V. Provenzano, R. D. Shull, I. Levin, E. D. Torre, and Y. Jin, J. Alloys Compd. 525, 34 (2012).

${ }^{15}$ T. Krenke, E. Duman, M. Acet, E. F. Wassermann, X. Moya, L. Mañosa, A. Planes, E. Suard, and B. Ouladdiaf, Phys. Rev. B 75, 104414 (2007).

${ }^{16}$ Z. D. Han, D. H. Wang, C. L. Zhang, S. L. Tang, B. X. Gu, and Y. W. Du, Appl. Phys. Lett. 89, 182507 (2006).

${ }^{17}$ J.-H. Chen, N. M. Bruno, I. Karaman, Y. Huang, J. Li, and J. H. Ross, Jr., Acta Mater. 105, 176 (2016).

${ }^{18}$ H. Wada, T. Morikawa, K. Taniguchi, T. Shibata, Y. Yamada, and Y. Akishige, Physica B 328, 114 (2003).

${ }^{19}$ L. Tocado, E. Palacios, and R. Burriel, J. Therm. Anal. Calorim. 84, 213 (2006).

${ }^{20}$ O. Tegus, E. Brück, K. H. J. Buschow, and F. R. de Boer, Nature 415, 150 (2002).

${ }^{21}$ F.-X. Hu, B.-G. Shen, J.-R. Sun, Z.-H. Cheng, G.-H. Rao, and X.-X. Zhang, Appl. Phys. Lett. 78, 3675 (2001).

${ }^{22}$ L. F. Bao, F. X. Hu, L. Chen, J. Wang, J. R. Sun, and B. G. Shen, Appl. Phys. Lett. 101, 162406 (2012).

${ }^{23}$ W. Bażela, A. Szytuła, J. Todorović, and A. Zięba, Phys. Status Solidi (a) 64, 367 (1981).

${ }^{24}$ V. Johnson and C. G. Frederick, Phys. Status Solidi (a) 20, 331 (1973).

${ }^{25}$ V. Johnson, Inorg. Chem. 14, 1117 (1975).

${ }^{26}$ J. Liu, Y. Si, Y. Gong, G. Xu, E. Liu, F. Xu, and D. Wang, J. Alloys Compd. 701, 858 (2017).

${ }^{\mathbf{2 7}}$ K. Morrison, A. Barcza, J. D. Moore, K. G. Sandeman, M. K. Chattopadhyay, S. B. Roy, A. D. Caplin, and L. F. Cohen, J. Phys. D 43, 195001 (2010).

${ }^{28}$ S. Anzai and K. Ozawa, Phys. Rev. B 18, 2173 (1978).

${ }^{29}$ W. Bażela, A. Szytuła, J. Todorović, Z. Tomkowicz, and A. Zięba, Phys. Status Solidi (a) 38, 721 (1976).

${ }^{30}$ H. Fjellvåg and A. F. Andresen, J. Magn. Magn. Mater. 50, 291 (1985).

${ }^{31}$ T. Kanomata, H. Ishigaki, T. Suzuki, H. Yoshida, S. Abe, and T. Kaneko, J. Magn. Magn. Mater. 140, 131-144 (1995).

${ }^{32}$ C. F. Sánchez-Valdés, J. L. S. Llamazares, H. F.-Z. Niga, D. Ríos-Jara, P. Alvarez-Alonso, and P. Gorria, Scr. Mater. 69, 211 (2013). 
${ }^{33}$ V. K. Sharma, M. A. Manekar, H. Srivastava, and S. B. Roy, J. Phys. D 49, 50LT01 (2016).

${ }^{34}$ J. B. A. Hamer, R. Daou, S. Özcan, N. D. Mathur, D. J. Fray, and K. G. Sandeman, J. Magn. Magn. Mater. 321, 3535 (2009).

${ }^{35}$ Y. K. Fang, C. C. Yeh, C. W. Chang, W. C. Chang, M. G. Zhu, and W. Li, Scr. Mater. 57, 453 (2007).

${ }^{36}$. Liu, K. Skokov, and O. Gutfleisch, Scr. Mater. 66, 642 (2012).

${ }^{37}$ C. L. Zhang, D. H. Wang, Q. Q. Cao, Z. D. Han, H. C. Xuan, and Y. W. Du, Appl. Phys. Lett. 93, 122505 (2008).

${ }^{38}$ N. T. Trung, L. Zhang, L. Caron, K. H. J. Buschow, and E. Brück, Appl. Phys. Lett. 96, 172504 (2010).

${ }^{39}$ J.-T. Wang, D.-S. Wang, C. Chen, O. Nashima, T. Kanomata, H. Mizuseki, and Y. Kawazoe, Appl. Phys. Lett. 89, 262504 (2006).

${ }^{40}$ E. K. Liu, W. Zhu, L. Feng, J. L. Chen, W. H. Wang, G. H. Wu, H. Y. Liu, F. B. Meng, H. Z. Luo, and Y. X. Li, Europhys. Lett. 91, 17003 (2010).

${ }^{41}$ S. Nizioł, A. Wesełucha, W. Bażela, and A. Szytuła, Solid State Commun. 39, 1081 (1981)

${ }^{42}$ S. Lin, O. Tegus, E. Bruck, W. Dagula, T. J. Gortenmulder, and K. H. J. Buschow, IEEE Trans. Magn. 42, 3776 (2006).

${ }^{43}$ X. Si, Y. Liu, X. Ma, J. Lin, J. Yang, and T. Zhou, J. Mater. Sci. 54, 3196 (2019).

${ }^{44}$ A. Aryal, A. Quetz, S. Pandey, T. Samanta, I. Dubenko, M. Hill, D. Mazumdar, S. Stadler, and N. Ali, J. Alloys Compd. 709, 142 (2017).

${ }^{45}$ N. T. Trung, V. Biharie, L. Zhang, L. Caron, K. H. J. Buschow, and E. Brück, Appl. Phys. Lett. 96, 162507 (2010).

${ }^{46}$ J. Torrens-Serra, C. A. Biffi, R. Santamarta, V. Recarte, J. I. Pérez-Landazábal, A. Tuissi, and E. Cesari, Mater. Charact. 93, 24 (2014).

${ }^{47}$ T. Samanta, I. Dubenko, A. Quetz, S. Stadler, and N. Ali, Appl. Phys. Lett. 101, 242405 (2012)

${ }^{48}$ S. Pal, C. Frommen, S. Kumar, B. Hauback, H. Fjellvåg, T. Woodcock, K. Nielsch, and G. Helgesen, J. Alloys Compd. 775, 22 (2019).

${ }^{49}$ Z. Wang, P. Xiu, L. Huang, Z. Nie, J. Zeng, D. E. Brown, Y. Ren, and Y. Wang, J. Magn. Magn. Mater. 406, 179 (2016).

${ }^{50} \mathrm{Q}$. Y. Ren, W. D. Hutchison, J. L. Wang, A. J. Studer, M. F. M. Din, S. M. Pérez, J. M. Cadogan, and S. J. Campbell, J. Phys. D 49, 175003 (2016).

${ }^{51}$ K. Ozono, Y. Mitsui, M. Hiroi, R. Y. Umetsu, K. Takahashi, K. Matsubayashi, Y. Uwatoko, and K. Koyama, Mater. Trans. 57, 316 (2016).

${ }^{52}$ S. Yuce, E. K. Doğan, B. Emre, N. M. Bruno, I. Karaman, and H. Yurtseven, J. Supercond. Nov. Magn. 30, 3587 (2017).

${ }^{53}$ C. L. Zhang, H. F. Shi, E. J. Ye, Y. G. Nie, Z. D. Han, and D. H. Wang, J. Alloys Compd. 639, 36 (2015).

${ }^{54}$ S. C. Ma, Y. X. Zheng, H. C. Xuan, L. J. Shen, Q. Q. Cao, D. H. Wang, Z. C. Zhong, and Y. W. Du, J. Magn. Magn. Mater. 324, 135 (2012).

${ }^{55} \mathrm{G}$. Meng, O. Tegus, W. Zhang, L. Song, and J. Huang, J. Alloys Compd. 497, 14 (2010).

${ }^{56}$ X. Si, Y. Shen, X. Ma, S. Chen, J. Lin, J. Yang, T. Gao, and Y. Liu, Acta Mater. 143, 306 (2018).
57. Zeng, Z. Wang, Z. Nie, and Y. Wang, Intermetallics 52, 101 (2014).

${ }^{58}$ K. Ozono, Y. Mitsui, R. Y. Umetsu, M. Hiroi, K. Takahashi, and K. Koyama, AIP Conf. Proc. 1763, 020003 (2016).

${ }^{59}$ T. Samanta, I. Dubenko, A. Quetz, S. Stadler, and N. Ali, J. Appl. Phys. 113, 17A922 (2013)

${ }^{60}$ D. Choudhury, T. Suzuki, Y. Tokura, and Y. Taguchi, Sci. Rep. 4, 7544 (2014).

${ }^{61}$ D. Zhang, Z. Nie, Z. Wang, L. Huang, Q. Zhang, and Y. Dong Wang, J. Magn. Magn. Mater. 387, 107 (2015).

${ }^{62}$ T. Gao, M. Wu, N. Qi, T. Zhou, X. Luo, Y. Liu, K. Xu, V. V. Marchenkov, H. Dong, Z. Chen, and B. Chen, J. Alloys Compd. 753, 149 (2018).

${ }^{63}$ K. A. Gschneidner, Jr. and V. K. Pecharsky, Annu. Rev. Mater. Sci. 30, 387 (2000).

${ }^{64}$ L. D. Griffith, Y. Mudryk, J. Slaughter, and V. K. Pecharsky, J. Appl. Phys. 123, 034902 (2018).

${ }^{65}$ T. Samanta, D. L. Lepkowski, A. Us Saleheen, A. Shankar, J. Prestigiacomo, I. Dubenko, A. Quetz, I. W. H. Oswald, G. T. McCandless, J. Y. Chan, P. W. Adams, D. P. Young, N. Ali, and S. Stadler, Phys. Rev. B 91, 020401(R) (2015).

${ }^{66}$ J.-H. Chen, A. Us Saleheen, S. K. Karna, D. P. Young, I. Dubenko, N. Ali, and S. Stadler, J. Appl. Phys. 124, 203903 (2018).

${ }^{67}$ J.-H. Chen, T. Poudel Chhetri, A. Us Saleheen, D. P. Young, I. Dubenko, N. Ali, and S. Stadler, Intermetallics 112, 106547 (2019).

${ }^{68}$ A. C. Larson and R. B. Von Dreele, "General structure analysis system (GSAS),” Report LAUR 86-748 (Los Alamos National Laboratory, 2004).

${ }^{69}$ B. H. Toby, J. Appl. Cryst. 34, 210 (2001).

${ }^{70}$ H. M. Rietveld, J. Appl. Cryst. 2, 65 (1969).

${ }^{71}$ J. C. Slater, J. Chem. Phys. 41, 3199 (1964).

${ }^{72}$ A. H. Morrish, The Physical Principles of Magnetism (John Wiley \& Sons, Inc., 2001), Chap. 7, p. 394.

${ }^{73}$ B. D. Cullity and C. D. Graham, Introduction to Magnetic Materials 2 nd ed. (John Wiley \& Sons, Inc., 2011), Chap. 9, p. 325.

${ }^{74}$ T. Kanomata, H. Ishigaki, K. Sato, M. Sato, T. Shinohara, F. Wagatsuma, and T. Kaneko, J. Magn. Soc. Jpn. 23, 418 (1999).

${ }^{75}$ S. Kaprzyk and S. Nizioł, J. Magn. Magn. Mater. 87, 267 (1990).

${ }^{76}$ S. Niziol, A. Zieba, R. Zach, M. Baj, and L. Dmowski, J. Magn. Magn. Mater. 38, 205 (1983).

${ }^{\mathbf{7 7}}$ A. M. Tishin and Y. I. Spichkin, The Magnetocaloric Effect and its Applications, Condensed Matter Physics (CRC Press, LLC, 2016).

${ }^{78}$ J.-H. Chen, A. Us Saleheen, P. W. Adams, D. P. Young, N. Ali, and S. Stadler, J. Appl. Phys. 123, 145101 (2018).

${ }^{79}$ L. Caron, Z. Q. Ou, T. T. Nguyen, D. T. C. Thanh, O. Tegus, and E. Brück, J. Magn. Magn. Mater. 321, 3559 (2009).

${ }^{80}$ Estimated by the product of $\Delta S_{\max }$ and the full width at half maximum of the entropy $\delta_{F W H M}:^{63,64}$

$$
R C P=\Delta S_{\max } \times \delta_{F W H M} .
$$

